\title{
The effect of triamcinolone in liposomes on oral wound healing in rats
}

\author{
Vladimira Erjavec ${ }^{1}$, Zlatko Pavlica ${ }^{1}$, Tomáš Fichtel ${ }^{2}$, Milan Petelin ${ }^{3}$ \\ ${ }^{1}$ University of Ljubljana, Veterinary Faculty, Small animal clinic, Ljubljana, Slovenia, \\ ${ }^{2}$ University of Veterinary and Pharmaceutical Sciences Brno, Faculty of Veterinary Medicine, \\ Small Animal Clinic, Brno Czech Republic \\ ${ }^{3}$ University of Ljubljana, Faculty of Medicine, Department of Oral Medicine and Periodontology, \\ Ljubljana, Slovenia
}

Received July 23, 2018

Accepted April 23, 2019

\begin{abstract}
The purpose of this study was to determine whether oral mucosa wounds in rats can be successfully treated with triamcinolone acetonide (TA), incorporated into liposomes. A round wound was inflicted on the oral mucosa of female Wistar rats divided into four groups of 12 animals. This wound was treated topically from day 1 with liposomes without the inclusion of TA and liposomes containing $0.01 \%$ or $0.05 \%$ TA. The wounds of the animals in the control group were not treated. Polymethyl metacrylate was used as an ointment for mixing in liposomes. The size of the wound was measured until day 6 . The area of inflammatory infiltrate under the wound was evaluated by histopathology, the expression of iNOS (inducible nitric oxide synthase) enzyme under the wound was evaluated by immunohistochemistry until day 6 . On the sixth day of experiment, the size of the wound and the area of the inflammatory infiltrate was the smallest in the group receiving empty liposomes (EL). Expression of iNOS was the most reduced in the group receiving EL. We conclude that oral mucosa wounds can be successfully treated with liposomes, although the incorporated drug triamcinolone would not be the appropriate drug for treating wounds of traumatic origin.
\end{abstract}

\section{Corticosteroid therapy, oral mucosa}

In the oral mucosa, inflammatory, atrophic, and ulcerative wounds of mainly unknown origin occur frequently (Lozada-Nur et al. 1994; Gonzalez-Moles and Scully 2005a,b), posing a major challenge for human oral medicine. These wounds are chronic, rarely spontaneously remitting, causing intense pain, and interfering with the daily activities and quality of life of the patients (Lozada-Nur et al. 1991, Gonzalez-Moles et al. 2002a,b, Gonzalez-Moles et al. 2003). Many diseases are autoimmune; corticosteroid therapy is the treatment of choice, however, there are few evidence-based data for the proper use of these drugs (Gonzalez-Moles and Scully 2005a,b; Gonzalez-Moles 2010). Despite the serious adverse effects associated with the use of these drugs, until recently it was necessary to prescribe systemic corticosteroids to control severe erosive oral diseases (Lozada et al. 1984; Gonzalez-Moles and Scully 2005a,b). When signs are limited to oral mucosa, these diseases can be controlled by topical corticosteroids which have proved to be highly efficacious and to cause fewer adverse effects compared to systemic corticosteroids (Gonzalez-Moles and Scully 2005a). Moist environment and constant activities of the facial, oral, and oropharyngeal musculature (Ten Cate 1998) contribute to the difficulties of maintaining a long-term contact of a drug to the oral mucosa, precluding a good control which is critical to the success of therapy (Thorburn and Fergus on 1994; Gonzalez-Moles et al. 2002b).

The ointment for active ingredients for oral disease treatment must therefore be able to adhere well to the mucosa (Bremecker et al. 1984). Liposomal formulations have been used to regulate the release of incorporated drugs, localizing the effect 
of the drugs. Treatment with liposomal formulations resulted in an increase in local and a decrease in systemic drug concentration (Mazei and Gulasekharam 1982; Harsanyi et al. 1986).

In inflammatory processes, nitric oxide (NO) has been shown to play an important role as a mediator of macrophage and neutrophil function (Moncada and Higgs 1993). The expression of inducible nitric oxide synthase (iNOS) occurs in conditions of inflammation, producing large amounts of NO. While the production of NO at normal rates provides the biological activity of this molecule, overproduction of NO through the iNOS pathway is increased in inflammatory diseases (Lappin et al. 2000; Keklikoglu et al. 2008).

The aim of this study was to investigate oral wound healing with the use of topical corticosteroid incorporated in liposomes.

\section{Materials and Methods}

The study protocol was submitted and approved by the Ethics Committee of the Veterinary Administration of the Republic of Slovenia ( $\left.\mathrm{N}^{\circ} 323-02-76 / 01\right)$.

Animals, production of oral wound and wound treatment

Female Wistar Hannover rats, weighing 200-220 g, were purchased from the Medical Experimental Department, Faculty of Medicine in Ljubljana, Slovenia. During the experiment, animals had free access to food and water. Before inflicting wounds, rats were anaesthetised by intraperitoneal injection of a mixture containing xylazine hydrochloride, 5-10 mg/kg (Rompun, Bayer, Leverkusen, Germany) and ketamine hydrochloride, 40-60 mg/kg (Ketanest 50, Parke-Davis, Berlin, Germany). Local anaesthetic lidocain (Xylocaine 10\% pump spray, AstraZeneca AB, Södertalje, Sweden) was applied to the buccal mucosa on the right and left cheek, then the buccal surface was exposed for $1 \mathrm{~s}$ to an iron circle of $3 \mathrm{~mm}$ in diameter, previously heated on the gas burner for $4 \mathrm{~s}$. This produced an immediate mucosal burn followed by development of a circular wound with a welldefined crater. One day after wound infliction (designated as experimental day 1) the rats were randomly divided into four groups of 12 animals:

group 1 - wounds were not treated, physiological healing was observed;

group 2 - wounds were treated with liposomes without the inclusion of the drug;

group 3 - wounds were treated with liposomes with $0.01 \%$ concentration of triamcinolone acetonide (TA);

group 4 - wounds were treated with liposomes with $0.05 \%$ concentration of TA.

Polymethyl metacrylate (PMM) was prepared as described by Sveinsson and Holbrook (1993) and Petelin et al. (1998). All liposomes were mixed with PMM at a ratio of 2:1, $40 \mu \mathrm{l}$ of mixture was applied with a pipette 4 times daily on each wound. Animals were not sedated. On the second, third, fourth and sixth day of the experiment, three animals from each group were euthanized.

Preparation of liposomes with encapsulated TA

Liposomes were prepared by the thin film method from cholesterol and hydrogenated soy lecithin (HSL) at a weight ratio of 3:7. The lipophilic phase containing phospholipid together with cholesterol and TA(Poly Chemicals, Varese, Italy) was dissolved in chloroform:methanol $(1: 1, \mathrm{v} / \mathrm{v})$. The solvent was removed in a rotary evaporator to give a thin film on the wall. The remaining solvent was removed completely under vacuum $\left(10\right.$ to $15 \mathrm{~min}$ at $40{ }^{\circ} \mathrm{C}$ and a pressure of $100 \mathrm{~Pa}$ ). The dry film was hydrated with distilled water at approximately $45^{\circ} \mathrm{C}$. The flask was shaken until the film was completely removed from the walls. The liposome dispersion was stabilized by stirring for $2 \mathrm{~h}$ on a magnetic stirrer (300 rpm) at room temperature. In the experiment, liposomes without TA and liposomes with $0.05 \%$ and $0.01 \%$ encapsulated TA were used. One $\mathrm{ml}$ of $0.01 \%$ HSL liposome dispersion contained $25 \mathrm{mg}$ of lipids and $1 \mathrm{mg}$ of TA; $1 \mathrm{ml}$ of $0.05 \%$ HSL liposome dispersion contained $25 \mathrm{mg}$ of lipids and $5 \mathrm{mg}$ of TA.

Evaluation of healing of the wounds

Quantitative healing of the wounds was assessed by photographing the wounds on anaesthetized animals by an intraoral digital camera (Planmeca, Intracam AF, Helsinki, Finland) on the first, second, third, fourth and sixth day of the experiment. Wounds were standardized, measured in $\mathrm{mm}^{2}$, and evaluated statistically using the computer program Image G (NIH, Bethesda, Maryland, USA).

Sample preparation, histological evaluation and evaluation of expression of iNOS in the wound

Rats were anaesthetised by intraperitoneal injection as described before and euthanized with an intracardial injection of $\mathrm{T} 61^{\mathrm{TM}}$ (Intervet international, Boxmeer, NL). The tissue with wound was excised from oral mucosa from the left and right cheek in each animal. Tissue samples were fixed in Bouin's solution for $24 \mathrm{~h}$, than placed in $70 \%$ alcohol until paraffin-embedded. Routine staining of tissue sections with haematoxylin and eosin was performed to evaluate the area of inflammatory infiltrate beneath the wound by light microscopy. For expression 
of iNOS, three sections of $7 \mu \mathrm{m}$ thickness were obtained by a standardized way from each paraffin block and stained by immunocytochemistry using a monoclonal antibody against iNOS. Tissue sections were mounted on APES (3-amino-propyl-tri-ethoxy silane, TESPA, Sigma, Taufkirchen, Germany) coated glass slides. Glass slides with tissue sections were dried overnight at a temperature of $50{ }^{\circ} \mathrm{C}$. Sections were dewaxed in xylene, rehydrated in alcohol solutions $(100 \%, 96 \%$ and $70 \%)$ and washed in $0.01 \mathrm{M}$ PBS (phosphate buffered saline ) with $0.05 \%$ Tween 20 . To block endogenous peroxidase activity, the glass slides were placed in $1 \%$ hydrogen peroxide for $20 \mathrm{~min}$ at room temperature, followed by washing in PBS-Tween 20. Normal goat serum diluted 1:5 in PBS-Tween 20 (Chemicon, Temecula, CA, USA) was used for $30 \mathrm{~min}$ for blocking to prevent the non-specific binding of primary antibody to the tissue proteins. Tissue sections were incubated over night at $4{ }^{\circ} \mathrm{C}$ with rabbit polyclonal antibodies against iNOS (Upstate, Lake Placid, NY, USA), diluted at 1:200 in PBS-Tween 20. Rabbit polyclonal antibodies IgG bind specifically with iNOS in examined tissue. Twenty-four hours later, the tissue slides were washed in PBS-Tween 20 ( 2 times for $5 \mathrm{~min}$ ) to remove residual primary antibody. Goat anti-rabbit antibodies IgG (Dako, Glostrup, Denmark), diluted at 1:100 in PBS, recognizing rabbit primary antibodies were added for $30 \mathrm{~min}$. The slides were washed in PBS-Tween 20 (2 times for $5 \mathrm{~min}$ ). Peroxidase-antiperoxidase complexes obtained from rabbit for determination of non-specific bound antibodies (Jackson Immunochemicals, West Grove, PA, USA), diluted at 1:500 in $0.05 \mathrm{M}$ Tris-HCl ( $\mathrm{pH} \mathrm{7.4)} \mathrm{were} \mathrm{added} \mathrm{for} 30 \mathrm{~min}$. Then, the slides were washed in PBS-Tween 20 two times for $5 \mathrm{~min}$. Finally, the slides were incubated with $0.05 \%$ (w/v) 3,3'diaminobenzidine tetrahydrochloride (Sigma, Germany) in $0.05 \mathrm{M}$ Tris- $\mathrm{HCl}\left(\mathrm{pH} 7.4\right.$ ) and $0.01 \% \mathrm{H}_{2} \mathrm{O}_{2}$ for 5-30 min and colour development was closely monitored. The enzyme reaction was stopped by rinsing the slide with distilled $\mathrm{H}_{2} \mathrm{O}$ two times for $5 \mathrm{~min}$. The slides were dehydrated with a second ethanol series $(70 \%, 96 \%$, in $100 \%)$ and xylene and covered with the cover slip.

Tissue slides were photographed by Sony 3CCD camera (Sony, Tokyo, Japan), mounted to the Nikon microphot FXA microscope (Nikon, Tokyo, Japan). Immunohistochemistry results of the staining were presented as a part of the immunopositive elements (cells) on a surface. All images were transformed to black and white and subjected to threshold transformation, controlling for the intensity of background staining. Threshold photographs were analysed using custom-made software Surfkvad (provided by Marko Kreft, MF, Ljubljana, Slovenia). Results are expressed as percentage of dark (stained) area in relation to the whole area of the microscopic field. Results were statistically analysed using Microsoft Excel software (SAS 9.01 procedure UNIVARIATE, MEANS, ANOVA in GLM (SAS Institute Inc., Cary, North Carolina, USA). The level of significance was $P<0.05$.

\section{Results}

\section{Oral mucosa wound healing}

The fastest and most effective wound healing was observed in the group treated with liposomes without TA. The baseline wound surface on day 0 was $7 \mathrm{~mm}^{2}$ in all groups. However, on the first day of experiment, the wound size was significantly greater in the group treated with liposomes with a $0.05 \%$ concentration of TA $(P<0.05)$.

Compared to the previous day, on the second day the wound increased in the group receiving liposomes with a $0.01 \%$ concentration of TA, and in the group without included $\mathrm{TA}$, the increase was significantly higher compared to the control group $(P<0.05)$. The size of the wound decreased in all groups on the third day and was smaller than on the first day. On the fourth day, in both groups receiving TA the size of the wound was significantly larger compared to the control group $(P<0.05)$. On the sixth day, the size of the wound in the control group and in the group receiving EL was significantly smaller compared to both groups receiving TA $(P<0.05$; Table 1$)$.

The wounds in both groups receiving TA also showed that the rate of epithelization was delayed till the sixth day compared to wound epithelization in the control group and in the group receiving EL.

\section{The area of inflammatory infiltrate in the oral wound in rat}

On the second day, the area of the inflammatory infiltrate was the largest in the group receiving liposomes with a $0.05 \%$ concentration of TA; the difference from the control group was significant $(P<0.05)$. Significantly the smallest inflammatory infiltrate was found in the group receiving liposomes without TA $(P<0.05)$.

In all groups, the area of inflammatory infiltrate was less extensive on the third day compared to the second day and was significantly smaller in the group receiving liposomes without TA compared to the control group $(P<0.05)$. In the group receiving liposomes 
Table 1. Size of the wound $\left(\mathrm{mm}^{2}\right)$ in the rat oral mucosa on different days over the 6 days of the experiment under different treatments.

\begin{tabular}{cccccc}
\hline Day & $\mathrm{N}$ & \multicolumn{1}{c}{$\mathrm{C}$} & $\mathrm{EL}$ & $0.01 \% \mathrm{TA}$ in $\mathrm{L}$ & $0.05 \% \mathrm{TA}$ in L \\
\hline 1 & 6 & $11.13 \pm 1.19$ & $11.71 \pm 0.93$ & $12.08 \pm 1.06$ & $13.15 \pm 0.58^{*}$ \\
2 & 6 & $10.58 \pm 0.92$ & $12.47 \pm 1.19^{*}$ & $12.81 \pm 1.05^{*}$ & $11.12 \pm 1.10$ \\
3 & 6 & $9.56 \pm 0.71$ & $10.41 \pm 1.09$ & $10.31 \pm 0.79$ & $10.03 \pm 0.90$ \\
4 & 6 & $9.63 \pm 0.96$ & $9.74 \pm 0.94$ & $12.71 \pm 0.75^{*}$ & $12.47 \pm 1.22^{*}$ \\
6 & 6 & $4.22 \pm 0.84$ & $3.49 \pm 0.86$ & $6.28 \pm 0.47^{*}$ & $7.03 \pm 1.25^{*}$ \\
\hline
\end{tabular}

Each value represents mean value $\pm \mathrm{SD}$ of six wound sizes on oral mucosa

* Significant difference in the wound size compared to the control group on the selected day $(P<0.05)$

$\mathrm{N}$ - number of wounds; $\mathrm{C}$ - control group; $\mathrm{EL}$ - liposomes from hydrogenated soy lecithin without inclusion of TA; TA - triamcinolone acetonide; $0.01 \%$ TA in L - liposomes from hydrogenated soy lecithin with $0.01 \%$ concentration of TA, $0.05 \%$ TA in $\mathrm{L}$ - liposomes from hydrogenated soy lecithin with $0.05 \%$ concentration of TA

with a $0.05 \%$ concentration of TA, the infiltrate was significantly more extensive than in the control group $(P<0.05)$ (Plate $\mathrm{X}$, Fig. 1).

On the fourth day, the area of inflammatory infiltrate was significantly larger in the group receiving $0.05 \%$ TA compared to the control group $(P<0.05)$. On the sixth day, the area of inflammatory infiltrate was decreased compared to the previous day (Table 2 ).

Table 2. The area of the inflammatory infiltrate $\left(\mathrm{mm}^{2}\right)$ beneath the oral wound in rats on different days of the experiment under different treatments.

\begin{tabular}{cccccc}
\hline Day & $\mathrm{N}$ & $\mathrm{C}$ & $\mathrm{EL}$ & $0.01 \%$ TA in $\mathrm{H}$ & $0.05 \%$ TA in L \\
\hline 2 & 6 & $6.94 \pm 0.99$ & $5.38 \pm 0.57^{*}$ & $7.91 \pm 0.74$ & $8.50 \pm 0.40^{*}$ \\
3 & 6 & $5.42 \pm 0.43$ & $4.35 \pm 0.19^{*}$ & $6.08 \pm 0.80$ & $6.32 \pm 0.61^{*}$ \\
4 & 6 & $4.56 \pm 0.56$ & $4.38 \pm 0.47$ & $4.97 \pm 0.20$ & $6.42 \pm 0.89 *$ \\
6 & 6 & $3.65 \pm 0.54$ & $2.93 \pm 0.42$ & $3.72 \pm 1.01$ & $4.41 \pm 0.83$ \\
\hline
\end{tabular}

Each value represents mean value $\pm \mathrm{SD}$ of the area of inflammatory infiltrate in six wounds on oral mucosa

* Significant difference in the area of inflammatory infiltrate compared to the control group on the selected day $(P<0.05)$

$\mathrm{N}$ - number of wounds; $\mathrm{C}$ - control group; EL - liposomes from hydrogenated soy lecithin without inclusion of TA; TA - triamcinolone acetonide; $0.01 \%$ TA in L - liposomes from hydrogenated soy lecithin with $0.01 \%$ concentration of TA; $0.05 \%$ TA in $\mathrm{L}$ - liposomes from hydrogenated soy lecithin with $0.05 \%$ concentration of TA.

\section{iNOS expression in the oral wound in rats}

On the second day of the experiment, the iNOS expression was significantly lower in the group receiving $0.01 \%$ TA compared to the control group $(; P<0.05)$.

Over the third, fourth and sixth day of the experiment, it was found that the expression of iNOS was reduced in all groups, except in the group receiving $0.01 \%$ concentration of TA. In that group, the expression of iNOS was increased on the third day (Plate X, Fig. 2). Over all the successive days, it was most reduced in the group receiving liposomes without TA, the expression was significantly lower on the third $(P<0.05)$ and sixth day $(P<0.05)$ compared to the control group (Table 3).

\section{Discussion}

In our previous study, we found that for the same effect, several-fold concentration of a free drug was needed in comparison to the concentration of a drug incorporated in liposomes (Erjavec et al. 2006). We also found that among the liposomes investigated, 
Table 3. Inducible nitric oxide synthase (iNOS) expression in oral wounds in rats on different days of the experiment under different treatments.

\begin{tabular}{cccccc}
\hline Day & $\mathrm{N}$ & \multicolumn{1}{c}{$\mathrm{C}$} & $\mathrm{EL}$ & $0.01 \%$ TA in L & $0.05 \%$ TA in L \\
\hline 2 & 6 & $12.93 \pm 3.08$ & $10.87 \pm 1.07$ & $9.28 \pm 2.37^{*}$ & $13.26 \pm 1.73$ \\
3 & 6 & $9.58 \pm 1.06$ & $6.18 \pm 1.54^{*}$ & $10.77 \pm 1.89$ & $10.42 \pm 1.57$ \\
4 & 6 & $5.60 \pm 1.12$ & $4.65 \pm 1.22$ & $7.52 \pm 1.19^{*}$ & $7.29 \pm 0.99^{*}$ \\
6 & 6 & $4.63 \pm 1.19$ & $2.66 \pm 0.87^{*}$ & $5.49 \pm 1.24$ & $6.48 \pm 1.0$ \\
\hline
\end{tabular}

Each value represents mean value \pm SD of the expression of iNOS in six wounds on oral mucosa

* Significant difference in the expression of iNOS compared to the control group on the selected day $(P<0.05)$

$\mathrm{N}$ - number of wounds; $\mathrm{C}$ - control group; $\mathrm{EL}$ - liposomes from hydrogenated soy lecithin without inclusion of TA; TA - triamcinolone acetonide; $0.01 \%$ TA in L - liposomes from hydrogenated soy lecithin with $0.01 \%$ concentration of TA; $0.05 \%$ TA in $\mathrm{L}$ - liposomes from hydrogenated soy lecithin with $0.05 \%$ concentration of TA.

multi-lamellar liposomes made from hydrogenated soy lecithin were the most effective carriers. The effectiveness of drug action was increased compared to a population of uni-lamellar liposomes. Uni-lamellar liposomes are smaller and more homogeneous liposomes with fewer layers or presumably with only one layer in their structure which break down on the surface of the oral mucosa at the same time, releasing the whole amount of the drug more uniformly and rapidly, and therefore having a shorter effect (Sentjurc et al. 1999; Krzic et al. 2001; Kristl et al. 2003; Erjavec et al. 2006). The population of non-extruded liposomes is very heterogeneous in size and lamellar structure; therefore, they release the entrapped substance more evenly over a prolonged period which is why they were used in this experiment.

The environment in the oral cavity means that traditional creams and ointments do not adhere adequately to the mucosal surface. The application of ointments with adhesive properties can enhance the retention time of the formulation at the site of action (Sveinsson and Holbrook 1993; Thorburn and Ferguson 1994) and also control the release rate of the drug. Adhesive contact to the mucosa is established by using mucoadhesive polymers as excipients (Duchene et al. 1988).

It has been found that among the different hydrophilic polymers, PMM is the most appropriate mucoadhesive ointment for local liposome application in oral cavity. Liposomes are most stable in this polymer and penetration of incorporated substance into the oral mucosa or gingiva was the most enhanced when PMM was used (Petelin et al. 1998). Therefore, PMM was chosen as the vehicle for the application of liposomes with and without the entrapped corticosteroid TA.

Severe erosive lesions of the oral mucosa have been classically treated with systemic corticosteroids. The frequency and severity of the adverse effects associated with the use of systemic corticosteroids have led to the increased prescription of topical corticosteroids for treating these pathologies. Topical corticosteroids are therefore some of the most common drugs used in oral pathology for treating atrophic erosive lesions that affect the mucosa. Nevertheless, a scientific body of evidence for their use in the oral cavity is virtually non-existent, and therefore many of the protocols followed are drawn from their use in dermatological setting (Scully et al. 1998).

Although TA is one of the most commonly used topical corticosteroids in oral pathology, there are very few published reports on its efficacy (Gonzalez-Moles and Scully $2005 \mathrm{a}, \mathrm{b})$. It is considered to be an average strength topical corticosteroid that has been effective in alleviating the signs and symptoms of many oral inflammatory conditions (Nicolazzo et al. 2005).

Studies on the subject recommend concentrations that range between $0.05 \%$ and $0.5 \%$. It is necessary to apply the medicine 3-10 times per day and for a period of 3 to 5 min each 
time in order to achieve results (Lozada-Nur et al. 1991; Lozada-Nur and Miranda 1997). In contrast, some authors used $0.2 \%$ TA in aqueous solutions and reported resolution of symptoms in $67 \%$ of their patients (Vincent et al. 1990). Others reported positive effects of TA in the treatment of oral lichen planus (Gonzalez-Garcia et al. 2006).

\section{Oral mucosa wound healing}

An enhanced effect of TA incorporated in HSL and therefore, a faster healing of wounds treated with TA, was expected. However, in this study, wounds treated with liposomes without the inclusion of TA healed the fastest.

The main provoking factor that induces inflammation of the gingival tissue and oral (buccal) mucosa is the presence of a variety of organisms in the microenvironment of the oral cavity that adhere and represent the bacterial biofilm on the teeth, gingival interfaces, tongue and the buccal mucosa (Teng 2003). Each site has a unique way of allowing the organisms to establish their residency. Normal flora in healthy individuals maintains similar patterns. When a local or systemic disease process or concomitant use of medications alter this pattern, atypical organisms begin to predominate and some normal organisms of benign nature become pathogenic (Little et al. 2002).

The products of biofilm bacteria, such as lipopolysaccharide molecules, are known to initiate a chain of reactions in the tissue leading to the host response as well as a destructive process (Haffajee and Socransky 1994).

The surface of the wound in both groups receiving TA was covered by a thick biofilm, which was not quantitatively evaluated in this study. Biofilm is composed of dead cells and fibrin, transudate and extracellular matrix, presenting desirable environment for secondary bacterial colonization. Both Gram-positive and Gram-negative organisms thrive within the biofilm and may penetrate and invade the submucosa.

In this study, liposomes with incorporated TA caused delayed wound healing and tissue regeneration. Higher concentration of TA caused slower healing which is in accordance with our previous findings (Erjavec et al. 2006) that show that liposomes allow controlled and continuous release of drug over a longer period of time.

The fastest healing of wound observed in the group receiving EL could be explained by the fact that liposomes with PMM adhered to the wound which played a role of wound dressing and prevented the adhesion of bacterial plaque which would modulate inflammation locally. The layer of PMM and liposomes also protected the wound against mechanical irritation.

\section{The area of inflammatory infiltrate}

The increased numbers of neutrophils which were in part the result of bacterial invasion and in part by impeded apoptosis were presumably caused by TA. In both groups treated with TA, it was detrimental to wound healing. It is well known that corticosteroids do not prevent infection and are contraindicated in the presence of bacterial infections and during inflammation therapy (Streeten and Phil 1975).

iNOS expression in oral wounds in rats

Corticosteroids inhibit the expression of various inflammatory genes including iNOS, which was expected in this study but not proved. The NO, synthetized by iNOS, is increased in many inflammatory diseases i.e. oral and leads to cellular injury. It is probable that bacterial plaque is responsible for NOS2 activation, since no NOS2 activity is found in the gingival tissues of sterile animals (Lohinai et al. 2001). Although NO may be produced in an attempt to kill plaque bacteria, it is likely that concentrations produced result in host tissue damage instead (Lohinai et al. 1998; Brennan et al. 2003). Further work is clearly needed in this area. 
In conclusion, we assume that due to the action of TA, a concomitant mucosal infection developed. Histological examination of the wounds showed no epithelial lining on the mucosal surface, beneath which we found damaged collagen fibres, dilated blood vessels and inflammatory infiltrate where neutrophils prevailed.

We must stress that animals in this experiment were not immunocompromised and therefore not an ideal model for investigation of vesiculo-erosive lesions in the oral cavity that are often the result of autoimmune diseases. The results show that PMM enhanced the residence time of liposomes on oral mucosa; in addition, TA included in liposomes achieved a greater effect, which in this study was delayed healing with wounds that were larger and slower to epithelialize.

Further studies are needed to investigate both effects of the incorporation of corticosteroids in liposomes for treatment of oral wounds in animals that are immunocompromised and of the incorporation of an appropriate antibacterial or antifungal agent for treatment of oral wounds caused by bacteria or fungi.

\section{Acknowledgements}

The study was supported by the Slovenian Research Agency, grant No. P4-0053. The authors would also like to thank Dr. Monika Zužek and Dr. Tomaž Büdefeld for providing help during the research.

\section{References:}

Bremecker KD, Strempel H, Klein G 1984: Novel concept for a mucosal adhesive ointment. J Pharm Sci 73: 548-552

Brennan PA, Thomas GJ, Langdon JD 2003: The role of nitric oxide in oral diseases. Arch Oral Biol 48: 93-100

Duchene DE, Touchard F, Pappas NA 1988: Pharmaceutical and medical aspects of bioadhesive systems for drug administration. Drug Dev Ind Pharm 14: 283-318

Erjavec V, Pavlica Z, Sentjurc M, Petelin M 2006: In vivo study of liposomes as drug carriers to oral mucosa using EPR oximetry. Int J Pharm 307: 1-8

Gonzalez-Garcia A, Diniz-Freitas M, Gandara-Vila P, Blanco-Carrion A, Garcia-Garcia A, Gandara-Rey J 2006: Triamcinolone acetonide mouth rinses for treatment of erosive oral lichen planus: efficacy and risk of fungal over-infection. Oral Dis 12: 559-565

Gonzalez-Moles MA 2010: The use of topical corticoids in oral pathology. Med Oral Patol Oral Cir Bucal 15: e827-e8231

Gonzalez-Moles MA, Morales-Garcia P, Rodriguez-Archilla A 2002a: Clobetasol mouthwash is effective for severe oral erosive lesions. Dent Abstr 47: 268

Gonzalez-Moles MA, Morales P, Rodriguez-Archilla A, Isabel IR, Gonzalez-Moles S 2002b: Treatment of severe chronic oral erosive lesions with clobetasol propionate in aqueous solution. Oral Surg Oral Med Oral Pathol Oral Radiol Endod 93: 264-270

Gonzalez-Moles MA, Ruiz-Avila I, Rodriguez-Archilla A, Morales-Garcia P, Mesa-Aguado F, BasconesMartinez A, Bravo M 2003: Treatment of severe erosive gingival lesions by topical application of clobetasol propionate in custom trays. Oral Surg Oral Med Oral Pathol Oral Radiol Endod 95: 688-692

Gonzalez-Moles MA, Scully C 2005a: Vesiculo-erosive oral mucosal disease management with topical corticosteroids: (1) fundamental principles and specific agents available. J Dent Res 84: 294-301

Gonzalez-Moles MA, Scully C 2005b: Vesiculo-erosive oral mucosal disease management with topical corticosteroids: (2) Protocols, monitoring of effects and adverse reactions, and the future. J Dent Res 84: 302-308

Haffajee AD, Socransky SS 1994: Microbial etiological agents of destructive periodontal diseases. Periodontol 2000 5: 78-111

Harsanyi BB, Hilchie JC, Mezei M 1986. Liposomes as drug carriers for oral ulcers. J Dent Res 65: $1133-1141$

Keklikoglu N, Koray M, Kocaelli H, Akinci S 2008: iNOS expression in oral and gastrointestinal tract mucosa. Dig Dis Sci 53: 1437-1442

Kristl J, Abramovic Z, Sentjurc M 2003: Skin oxygenation after topical application of liposome-entrapped benzyl nicotinate as measured by EPR oximetry in vivo: influence of composition and size. AAPS Pharm Sci 5: 19-27

Krzic M, Sentjurc M, Kristl J 2001: Improved skin oxygenation after benzyl nicotinate application in different carriers as measured by EPR oximetry in vivo. J Control Release 70: 203-211

Lappin DF, Kjeldsen M, Sander L, Kinane DF 2000: Inducible nitric oxide synthase expression in periodontitis. J Periodontal Res 35: 369-373

Little JW, Miller CS, Rhodus NL, Falace D (Eds) 2002: Dental Management of the Medically Compromised Patient. $6^{\text {th }}$ edn. St. Louis, Mosby. 
Lohinai Z, Benedek P, Fehér E, Györfi A, Rosivall L, Fazekas A, Salzman AL, Szabó C 1998: Protective effects of mercaptoethylguanidine, a selective inhibitor of inducible nitric oxide synthase, in ligature-induced periodontitis in the rat. Br J Pharmacol. 123: 353-360. Erratum in: Br J Pharmacol 123: 741

Lohinai Z, Stachlewitz R, Virag L, Szekely AD, Hasko G, Szabó C 2001: Evidence for reactive nitrogen species formation in the gingivomucosal tissue. J Dent Res 80: 470-475

Lozada-Nur F, Huang MZ, Zhou GA 1991: Open preliminary clinical trial of clobetasol propionate ointment in adhesive paste for treatment of chronic oral vesiculoerosive diseases. Oral Surg Oral Med Oral Pathol 71: 283-287

Lozada-Nur F, Miranda C 1997: Oral lichen planus: topical and systemic therapy. Semin Cutan Med Surg 16: 295-300

Lozada-Nur F, Miranda C, Maliksi R 1994: Double-blind clinical trial of 0.05\% clobetasol propionate ointment in orabase and $0.05 \%$ fluocinonide ointment in orabase in the treatment of patients with oral vesiculoerosive diseases. Oral Surg Oral Med Oral Pathol Oral Radiol Endod 77: 598-604

Lozada F, Silverman S Jr, Migliorati C 1984. Adverse side effects associated with prednisone in the treatment of patients with oral inflammatory ulcerative diseases. J Am Dent Assoc 109: 269-270

Mazei M, Gulasekharam V 1982: Liposomes: a selective drug delivery system for the topical route of administration. Gel dosage form. J Pharm Pharmacol 34: 473-474

Moncada S, Higgs A 1993. The L-arginine-nitric oxide pathway. N Engl J Med 329: 2002-2012

Nicolazzo JA, Reed BL, Finnin BC 2005: Enhancing the buccal mucosal uptake and retention of triamcinolone acetonide. J Control Release 105: 240-248

Petelin M, Sentjurc M, Stolic Z, Skaleric U 1998: EPR study of mucoadhesive ointments for delivery of liposomes into the oral mucosa. Int J Pharm 173: 193-202

Scully C, Beyli M, Ferreiro MC, Ficarra G, Gill Y, Griffiths M, Holmstrup P, Mutlu S, Porter S, Wray D1998: Update on oral lichen planus: etiopathogenesis and management. Crit Rev Oral Biol Med 9: 86-122

Sentjurc M, Vrhovnik K, Kristl J 1999: Liposomes as a topical delivery system: the role of size on transport studied by the EPR imaging method. J Control Release 59: 87-97

Streeten DH, Phil D 1975: Corticosteroid therapy. J Am Med Assoc 232: 944-948

Sveinsson SJ, Holbrook WP 1993: Oral mucosal adhesive ointment containing liposomal corticosteroid. Int J Pharm 95: 105-109

Ten Cate AR 1998: Oral histology: development, structure, and function. St. Louis: Mosby Year Book, pp. 345-385

Teng YT 2003: The role of acquired immunity and periodontal disease progression. Crit Res Oral Biol Med 14: 237-252

Thorburn DN, Ferguson MM 1994: Topical corticosteroids and lesions of the oral mucosa. Adv Drug Delivery Rev 13: 135-149

Vincent SD, Baker KA, Williams TP 1990: Oral lichen planus: the clinical, historical, and therapeutic features of 100 cases. Oral Surg Oral Med Oral Pathol Oral Radiol Endod 70: 165-171 
Plate X

Erjavec V. et al.: Effect of triamcinolone ... pp. 225-232
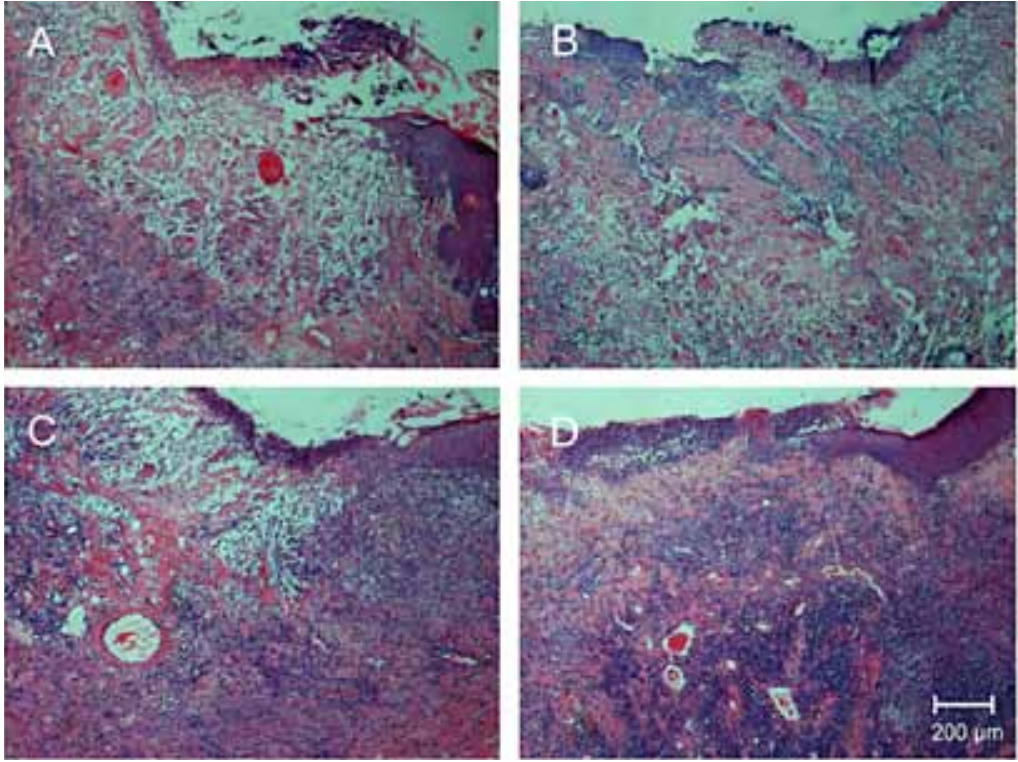

Fig. 1. Comparison of inflammatory infiltrate expression in an oral wound in the rat on the third day after the wound was inflicted and received different treatment

A - without treatment; B - liposomes from hydrogenated soy lecithin without triamcinolone acetonide (TA); C - liposomes with $0.01 \%$ concentration of TA; D - liposomes with $0.05 \%$ concentration of TA. Arrows pointing areas with inflammatory infiltrate. Scale on the picture D is the same for all four pictures.
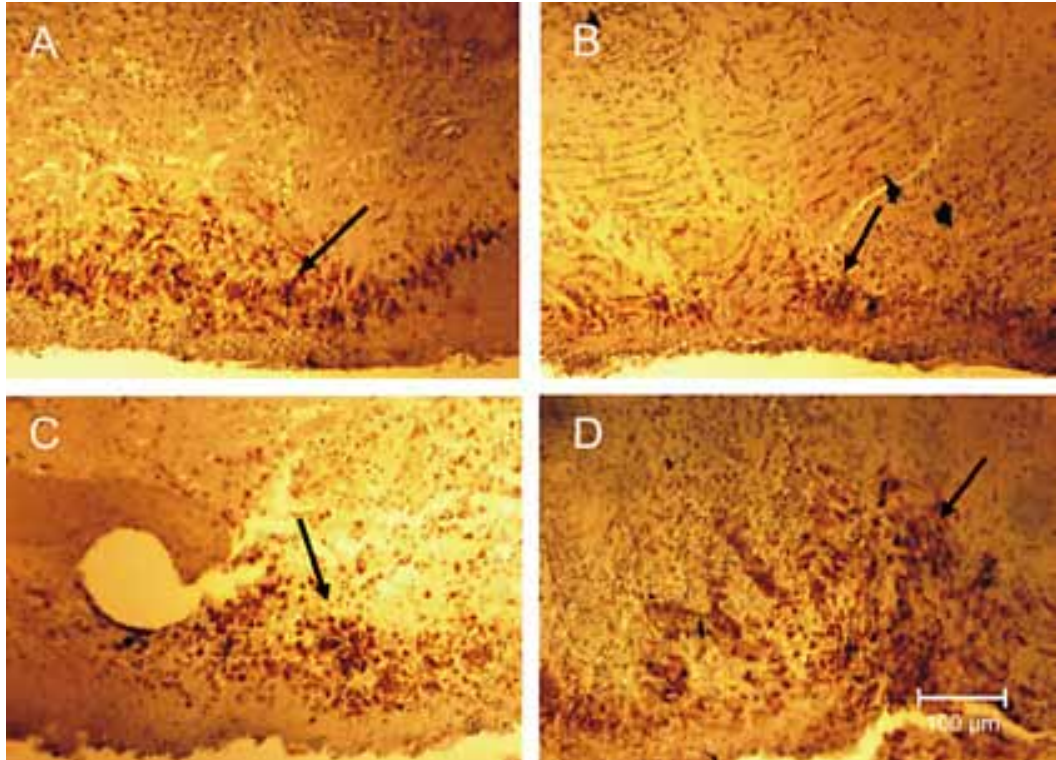

Fig. 2. Comparison of inducible nitric oxide synthase (iNOS) expression in oral wounds in rat on the third day after the wounds were produced and received different treatment

A - without treatment; B - liposomes from hydrogenated soy lecithin without triamcinolone acetonide (TA); C - liposomes with $0.01 \%$ concentration of TA; D - liposomes with $0.05 \%$ concentration of TA. Arrows pointing areas with iNOS expression. Scale on the picture D is the same for all 4 pictures. 